\title{
Obturación endodóntica de las raíces mesiales de molares inferiores con sellador Bio-C Sealer y AHPlus
}

\section{Endodontic obturation of mesial roots of mandibular molars with Bio-C Sealer and AHPlus}

Paula Alison Hernandez ${ }^{1}$, Enrique Fernández Bodereau ${ }^{2}$,Teresa Moyano ${ }^{1,2}$, Sandra Arguelles ${ }^{1}$, Catalina Solla $^{1}$, Facundo Mattea ${ }^{3,4}$, Mauro Valente ${ }^{5,6}$, Gabriela Martin ${ }^{1,7}$.

1 Universidad Católica de Córdoba. Facultad de Ciencias de la Salud. Carrera de Especialización de Endodoncia

2 Cátedra de Clínica de Prótesis y Carrera de Especialista en Prótesis e Implantología; Facultad de Odontología. Universidad Nacional de Córdoba.

3 Departamento de Química Orgánica, Facultad de Ciencias Químicas, Universidad Nacional de Córdoba

4 Instituto de Investigación y Desarrollo de Procesos en Ingeniería de Procesos y Química Aplicada.

5 Instituto de Física E. Gaviola - CONICET, \& Laboratorio de Investigación e Instrumentación en Física Aplicada a la Medicina e Imágenes por Rayos X, LIIFAMIRx, FAMAF, Universidad Nacional de Córdoba, Córdoba; Argentina.

6 Centro de Física e Ingeniería en Medicina, CFIM, \& Departamento de Ciencias Físicas, Universidad de La Frontera, Temuco; Chile.

7 Universidad Nacional de Córdoba. Facultad de Odontología. Cátedra de Endodoncia.

Correspondencia: Gabriela Martín. Universidad Católica de Córdoba. Carrera de Endodoncia, Córdoba, Argentina. Email: ggmartin@ hotmail.com

\section{Resumen}

La obturación del conducto radicular se realiza con conos de gutapercha y un sellador endodóntico, el cual rellena los espacios entre la pared dentinaria y el material obturador; y las irregularidades del conducto radicular. El sellador Bio-C Sealer es un cemento biocerámico con propiedades bioactivas, mientras que el sellador AH Plus es un cemento a base de resina. La hipótesis del presente estudio fue que el sellador BioC Sealer ocupa mayor volumen y superficie, respecto del sellador AH Plus.

OBJETIVO: Comparar el uso del sellador Bio-C Sealer con el sellador AH Plus en la obturación de conductos mesiales de molares inferiores.

MATERIALES Y MÉTODOS: Se seleccionaron 40 conductos (20 mesio-vestibulares y 20 mesiolinguales) de 20 raíces mesiales de molares inferiores permanentes. Los conductos fueron instrumentados con limas Wave One Gold Small (Dentsply, Maillefer) y WaneOne Gold Primary e irrigados con hipoclorito de sodio. Para la obturación, la muestra se dividió en dos grupos de 20 conductos cada uno, de acuerdo al sellador endodóntico utilizado. Grupo A: sellador AH Plus (Dentsply, Maillefer) y Grupo B: sellador Bio-C Sealer (Angelus). Finalizada la obturación, se utilizó la microtomografía computada (microCT) de alta resolución con un detector tipo flat-panel certificado de la compañía Varian R (USA) modelo PaxScan 2020+ para obtener 180 proyecciones tomográficas de cada muestra. Luego se reconstruyó la imagen 3D de la muestra utilizando algoritmos específicos dedicados en el software MatLab (MathWorks, USA), y con desarrollos propios del equipo de investigación en lenguaje Python en las instalaciones del LIIFAMIRx de la Universidad Nacional de Córdoba. Los datos obtenidos fueron analizados estadísticamente mediante el test de Mann Whitney.

RESULTADOS: No se encontraron diferencias significativas en el volumen de obturación entre las muestras tratadas con sellador AH Plus y sellador Bio C- Sealer ( $>>0,05)$. Respecto al volumen de espacios vacíos, la frecuencia de casos en los cuales pudieron detectarse volúmenes de vacíos mensurables $(\geq 0,01$ $\mathrm{mm} 3$ ) fue del 15\%; registrándose 2 en el grupo BIO-C sealer (5\%) y 4 en AH Plus (10\%). 
CONCLUSIÓN: Bajo las condiciones de este estudio, puede concluirse que ambos selladores AH Plus y Bio-C Sealer resultaron adecuados para la obturación de los conductos mesio-vestibulares y mesiolinguales de molares inferiores. Sin embargo, el volumen de espacios vacíos fue menor cuando se usó el sellador biocerámico Bio-C Sealer.

Palabras claves: Sellador endodóntico, obturación del conducto radicular, diente molar.

\begin{abstract}
Root canal obturations are usually performed with guttapercha cones and an endodontic sealer, filling the space between the dentinal wall and filling material, and the irregularities of the root canal. Bio-C Sealer is a bioceramic and bioactive sealer, whereas $\mathrm{AH}$ Plus is a resin endodontic sealer. The hypothesis of the present study was that Bio-C sealer occupies greater volume and surface than AH plus.

OBJECTIVE: To compare the use of the Bio-C Sealer and AH Plus in the filling of mesial root canals of mandibular molars.

MATERIALS AND METHODS: Forty root canals (20 mesio-buccal and 20 mesio-lingual) of 20 mesial roots of mandibular permanent molars were selected. The mesial root canals were instrumented with Wave One Gold Small files (Dentsply, Maillefer) and Wave One Gold Primary files and irrigated with sodium hypochlorite. After the instrumentation, root canals were irrigated with EDTA, as final irrigation, to induce sealer penetration through dentinal tubules. For the obturation, the samples were divided into two groups according to the used endodontic sealer. Group A: AH plus sealer (Dentsply, Maillefer) and Group B: BioC Sealer bioceramic sealer (Angelus). Afterwards, a high-resolution micro-tomography (micro-CT), with a certified flat-panel detector from Varian R (USA), model PaxScan 2020+ was used to obtain 180 tomographic projections of each sample. Then, 3D sample images were reconstructed by using specific dedicated algorithms in MatLab (MathWorks, USA), and specific coding developed in Python, at LIIFAMIRx - National University of Cordoba. Recorded data were statistically analyzed by the Mann Whitney Test.

RESULTS: No significant differences were found in the sealing volume between the AH Plus and the BioC Sealer $(p>0,05)$. A higher index of empty spaces was observed in the endodontic filling when AH Plus was used compared to Bio-C Sealer. Empty spaces were found in 15\% of root canals; $10 \%$ for AH Plus and $5 \%$ for Bio-C sealer.

CONCLUSION: Within the limitations of the current study, it can be concluded that both sealers, AH Plus and Bio-C Sealer, were adequate to fill mesial- buccal and mesial-lingual root canals of mandibular molars. Although, the volume of empty spaces was lower when Bio-C Sealer bioceramic was used.
\end{abstract}

KeyWords: endodontic filling, root canal obturation, molar tooth.

\title{
Introducción
}

El éxito en el tratamiento endodóntico depende de la prevención y el control de la infección del conducto radicular, que se logra mediante una limpieza, conformación y sellado adecuado. Una obturación deficiente podría conducir a un fracaso endodóntico, a pesar de la realización de una meticulosa preparación del conducto radicular ${ }^{1,2}$. El material más utilizado para la obturación del conducto radicular se basa en los conos de gutapercha, los cuales se fabrican con diferentes calibres y conicidad. Las discrepancias morfológicas entre los instrumentos y conos de gutapercha, sumada a la anatomía variada de los conductos radiculares, crean dificultades significativas para la obturación tridimensional del sistema de conductos ${ }^{3}$.

El molar inferior, no sólo es uno de los elementos dentarios más tratados endodónticamente, sino que también plantea una serie de desafíos anatómicos. Estas complicaciones incluyen conductos múltiples, istmos, conductos laterales y ramificaciones apicales ${ }^{4,5}$. El primer molar inferior presenta dos raíces, una mesial y otra distal, pero en ocasiones puede presentar tres raíces, con una tercera raíz distal llamada Radix. La raíz mesial, que es la más ancha de las dos, se curva en sentido mesial desde la línea cervical hasta el tercio medio de la raíz, y posteriormente, se angula en sentido distal hasta el ápice. Los conductos de la raíz mesial son: uno mesio-vestibular (MV) y el otro mesio-lingual (ML), los que suelen ser curvos, con una curvatura más pronunciada en el conducto MV en el plano vestíbulo- lingual (VL) ${ }^{6}$. Los conductos de las raíces distales son: el Distal (si sólo existe un conducto) y Disto-vestibular (DV) y Distolingual (DL) si existen varios conductos. El 
conducto distal es normalmente recto en todo su recorrido, de sección transversal oval o aplanada y más amplio que los mesiales. Frecuentemente, 1-2 $\mathrm{mm}$ apicales del conducto presentan una curvatura hacia el distal. El segundo molar inferior se caracteriza por la proximidad de sus raíces mesial y distal.

Debido a estas variables anatómicas, para lograr un sellado tridimensional en la obturación del conducto, es necesario usar conos de gutapercha con un sellador endodóntico ${ }^{3}$. Los selladores del conducto radicular son necesarios para rellenar el espacio entre la pared dentinaria y el material obturador. Además, ocupan los espacios vacíos y las irregularidades del conducto radicular, como así también, los conductos laterales, conductos accesorios, istmos y deltas apicales, que resultan difíciles de acceder en la instrumentación ${ }^{7}$. El material sellador, debe presentar adecuadas propiedades biológicas y fisicoquímicas, no debe irritar los tejidos perirradiculares, e idealmente debe estimular la reparación y sellado biológico por deposición de tejido mineralizado en el foramen apical. Además, debe presentar adhesividad y estabilidad dimensional para evitar la circulación de fluidos entre el conducto y el periápice $^{1,8}$.

Los selladores endodónticos más utilizados son aquellos a base de óxido de zinc y eugenol, hidróxido de calcio y resina ${ }^{9}$. El sellador AH Plus (Dentsply Maillefer, Ballaigues, Suiza) es un material a base de resina epoxi y el más utilizado debido a sus buenas propiedades físicas y químicas; y su adecuada capacidad de sellado ${ }^{10}$. Para casos específicos, como reabsorciones radiculares, perforaciones, apexificación y rellenos retrógrados, se desarrollaron nuevos materiales biocompatibles para mejorar el resultado clínico ${ }^{9}$.

Los biocerámicos están compuestos de óxido de cinc, silicatos cálcicos, fosfato cálcico monobásico, $\mathrm{Ca} \quad(\mathrm{OH}) 2$ y varios agentes obturadores y espesantes. Como selladores hidrofílicos, utilizan la humedad del conducto para completar la reacción de fraguado y no se contraen al fraguar. Son biocompatibles y tienen propiedades antimicrobianas ${ }^{8}$. El primer material biocerámico utilizado con éxito en endodoncia fue el cemento MTA (Agregado de Trióxido Mineral), desarrollado a base de cemento Portland ${ }^{11}$. Los productos originales de MTA fueron difíciles de manipular y relativamente gruesos, lo que impidió alcanzar el bajo espesor de película necesario para los selladores de conductos radiculares ${ }^{12}$. El color gris fue una desventaja para las propiedades estéticas del material, además de los riesgos percibidos asociados con la presencia de aluminio, arsénico y metales pesados en el cemento Portland. Es por ello, que se desarrolló una nueva familia de cementos a base de silicato de calcio más puro. El silicato tricálcico reemplazó a la formulación original del cemento Portland, eliminando el aluminio y los oligoelementos. Estos nuevos materiales fueron desarrollados basados en la química del radiopacificador y del silicato tricálcico13, demostrando ser más adecuados para su uso como selladores. Recientemente, se ha introducido selladores biocerámicos como EndoSequence BC Sealer (Brasseler USA, Savannah, GA) que utiliza la humedad inherentemente presente en los túbulos dentinarios para su reacción de fraguado ${ }^{14}$, como así también el sellador Bio-C Sealer (Angelus).

Uno los parámetros para evaluar las propiedades de un sellador es su capacidad para penetrar en los túbulos dentinarios, conductos laterales y delta apical $^{15}$. La penetración del sellador en los túbulos dentinarios de la dentina radicular forma una barrera física ${ }^{16}$, mejora la retención del relleno de la raíz ${ }^{17}$ y sepulta las bacterias residuales ${ }^{18}$. Cuanto mayor es la penetración de un sellador en los túbulos dentinarios, más se incrementa su efecto antibacteriano $^{19}$. Las propiedades físicas $\mathrm{y}$ químicas, como el tamaño de las partículas, la solubilidad, la viscosidad y la tensión superficial, influyen en la profundidad de penetración del material en el túbulo de la dentina ${ }^{18}$.

\section{Objetivo}

El objetivo del presente estudio fue comparar el uso del sellador Bio-C Sealer con el sellador AH Plus en la obturación de conductos mesiales de molares inferiores.

\section{Material y métodos}

\section{Tipo de Investigación: Experimental}

Características generales: La presente investigación se desarrolló en la Carrera de Endodoncia de la Facultad de Ciencias de la Salud de la Universidad Católica de Córdoba y en el Instituto de Física E. Gaviola - CONICET, Laboratorio de Investigación en Física Aplicada a la Medicina e Imágenes por Rayos X LIIFAMIRX - FaMAF, donde se llevó a cabo el estudio de microtomografía computada de rayos $\mathrm{X}$ de alta resolución.

Se seleccionaron 20 raíces mesiales de primeros y segundos molares inferiores permanentes humanos extraídos recientemente. Los mismos fueron desinfectados con hipoclorito de sodio $(\mathrm{NaOCl})$ al $5 \%$ durante 24 horas, y posteriormente fueron almacenados en solución salina para que preserven la humedad de los túbulos dentinarios de los dientes. 
Al momento de ser utilizados, se cubrieron las raíces con cinta de teflón, a fin de simular el tejido periodontal y se procedió a cortar la corona a $4 \mathrm{~mm}$ del cuello anatómico utilizando un disco de carborundum. Se tomaron radiografías en sentido orto-radial y próximo-proximal a una distancia estandarizada de 10 centímetros entre el extremo emisor de rayos $\mathrm{X}$ y el molar. Todas las radiografías iniciales previas se tomaron con radiovisiógrafo (Kodak Carestream Dental RVG 5200) para evaluar la anatomía interna de los conductos.

Para la preparación de la cavidad de acceso coronario, se talló el acceso inicial con fresa redonda \# 4 (Jota) y se rectificó el acceso con fresa Endo Z (Dentsply, Maillefer). Posteriormente, se realizó la limpieza y conformación del conducto radicular, para lo cual se irrigó la cámara pulpar con $5 \mathrm{~mL}$ de solución de NaOCL al $5.25 \%$; seguido de cateterismo del conducto con lima $\mathrm{K}$ \#10 (Dentsply, Maillefer). El acceso radicular y permeabilidad mecanizada de los conductos se realizó con lima Proglider (Dentsply, Maillefer). Se determinó la longitud de trabajo (LT) $1 \mathrm{~mm}$ más corto del foramen apical. Para la preparación de tercio apical, se usaron limas Wave One Gold Small (20.07 Dentsply, Maillefer) finalizando con Wave One Gold Primary (25.07). Finalmente, se usó una lima K \#10 para verificar la permeabilidad del conducto.

A cada cambio de instrumento se irrigó con $2 \mathrm{~mL}$ de hipoclorito de sodio al 5.25\%. Para la desinfección final, se irrigó con $2 \mathrm{~mL}$ de $\mathrm{NaOCl}$ al $5.25 \%$ activado 30 segundos con Endoactivator (Dentsply, Maillefer), seguido de $2 \mathrm{~mL}$ de ácido etilendiaminotetraacético (EDTA, Tedequim) al $17 \%$ activado 60 segundos con Endoactivator y se dejó actuar durante 2 minutos. Luego, se usaron 2 $\mathrm{mL}$ de hipoclorito de sodio al $2.5 \%$ activado durante 30 segundos con Endoactivator (Dentsply, Maillefer) y finalmente $2 \mathrm{~mL}$ de solución fisiológica. Finalmente, se secaró los conductos con puntas de papel estéril (Meta Biomed).

Para la obturación de los conductos radiculares, se seleccionó el cono de gutapercha maestro de acuerdo al último instrumento utilizado, y a la longitud de trabajo correspondiente a cada conducto. Se tomaron radiografías digitales para corroborar la correcta adaptación del mismo y la muestra fue dividida aleatoriamente en dos grupos de 10 molares cada uno:

-Grupo A: Sellador AH plus (Dentsply, Maillefer). -Grupo B: Sellador Bio-C Sealer (Angelus).

En el grupo A, los conductos fueron obturados con sellador AH plus que se mezcló en una proporción 1:1 con pasta de epóxido y amina hasta que se logró una mezcla homogénea. Se llevó el cono maestro al conducto junto con el sellador y se realizó la técnica de compactacón lateral.
En el grupo B, los conductos fueron obturados con sellador Bio-C Sealer (Angelus). Se llevó el sellador al conducto con la jeringa que contiene el sellador a $2 \mathrm{~mm}$ menos de la longitud de trabajo y posteriormente se llevó el cono maestro al conducto a LT junto con el sellador. Se realizó la técnica de cono único.

Finalizada la obturación, se utilizó la técnica de micro-tomografía computada de rayos X (microCT) de alta resolución utilizando un detector tipo "flat-panel" certificado, de la companía Varian R (USA) modelo PaxScan 2020+ para obtener 180 proyecciones tomográficas de cada muestra Luego se reconstruyó la imagen $3 \mathrm{D}$ de cada molar mediante algoritmos específico dedicados desarrollados en el software MatLab (MathWorks R, USA) con licencia oficial 3407-8985-43329223-7918 y con desarrollos propios del equipo de investigación en lenguaje Python, en las instalaciones del LIIFAMIRx y Laboratorio de Física Aplicada a la Medicina (LaFAM, FAMAF).

\section{Análisis estadístico}

Los datos obtenidos fueron analizados estadísticamente con el test de Mann Whitney, con un nivel de significación del $5 \%$.

\section{Consideraciones éticas}

El estudio se realizó cumpliendo con la normativa de la Declaración de Helsinki, Buenas Prácticas Clínicas de ANMAT y Ley Provincial de investigación en salud $\mathrm{N}^{\circ}$ 9694. Se asegura protección de datos personales de los pacientes según la Ley 25.326. Los autores declaran no tener conflictos de intereses.

\section{Resultados}

A partir de los datos densitométricos obtenidos en la microtomografía computada, se calculará los porcentajes de volumen de gutapercha, sellador y espacios vacíos de los conductos radiculares. En la (Tabla 1) se expresan los valores promedio de los porcentajes de la obturación de los conductos mesio-vestibulares y mesio-linguales obturados con Bio-C Sealer y AH Plus. 
Tabla 1. Media \pm desviación estándar de porcentajes de volúmenes ocupados por sellador, gutapercha y espacios vacíos según conducto y grupo de sellador.

\begin{tabular}{|c|c|c|c|c|c|c|c|c|c|c|c|}
\hline Conducto & GRUPO & $\mathrm{n}$ & \multicolumn{3}{|c|}{ Sellador } & \multicolumn{3}{|c|}{ Gutapercha } & \multicolumn{3}{|c|}{ Esp. vacios } \\
\hline \multirow{2}{*}{ MV } & $\begin{array}{l}\text { BIO-C } \\
\text { SEALER }\end{array}$ & 10 & 81,3 & \pm & 10,3 & 18,7 & \pm & 10,3 & 0,00 & \pm & 0,01 \\
\hline & AHPLUS & 10 & 78,6 & \pm & 9,4 & 21,4 & \pm & 9,4 & 0,03 & \pm & 0,09 \\
\hline \multirow{2}{*}{ ML } & $\begin{array}{l}\text { BIOCC. } \\
\text { SEALER }\end{array}$ & 10 & 81,6 & \pm & 6,1 & 18,4 & \pm & 6,1 & 0,00 & + & 0,00 \\
\hline & AHPLUS & 10 & 85,8 & \pm & 10,4 & 14,0 & \pm & 10,1 & 0,22 & \pm & 0,69 \\
\hline
\end{tabular}

La frecuencia de casos en los cuales pudo detectarse volúmenes de espacios vacíos mensurables $(\geq 0,01 \mathrm{~mm} 3)$ fue del $15 \%$; lo cual se observó en sólo 6 conductos radiculares, registrándose 2 en el grupo obturado con Bio-C Sealer (5\%) y 4 en el grupo de AH Plus (10\%). En la (Figura 1) se observa que el porcentaje de espacios vacíos de los conductos radiculares, en ambos grupos de estudio fue menor que la de sellador y gutapercha. Al aplicar el Test de MannWhitney, las diferencias no resultaron estadísticamente significativas entre ambos grupos de estudio $(\mathrm{p}>0,05)$.

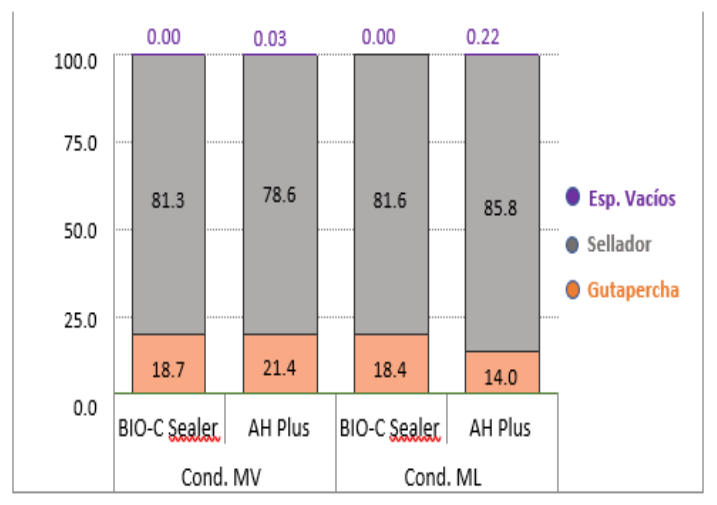

Figura 1. Porcentaje de volumen ocupado por gutapercha, sellador y espacios vacíos en los conductos mesio-vestibulares (MV) y mesio-linguales (ML) obturados con Bio-C Sealer y AH Plus.

\section{Discusión}

La obturación tridimensional es un desafío en la práctica clínica y tiene como objetivo evitar el desarrollo de espacios sin rellenar o burbujas que podrían generar condiciones favorables para el desarrollo bacteriano1. Una de las propiedades fundamentales del sellador es la fluidez, lo cual le permite al sellador llenar áreas de difícil acceso, como las irregularidades estrechas de la dentina, istmos, conductos accesorios y espacios vacíos entre los conos maestros y accesorios ${ }^{20}$. Se seleccionó como objeto de estudio el molar inferior, no sólo porque es uno de los elementos dentarios más tratados endodónticamente, sino que también plantea una serie de desafíos anatómicos. Los conductos radiculares MV y ML de la raíz mesial de los molares inferiores presenta una de las mayores variantes anatómicas, incluyendo conductos múltiples, istmos, conductos laterales y ramificaciones apicales.

En este estudio, la totalidad de la muestra fue instrumentada con limas Wave One Gold Small (20.07) y Primary (25.07), minimizando de esta manera, las posibles variables como la anatomía dentaria, el tamaño del conducto y el diámetro del foramen apical. Además, todos los molares fueron irrigados bajo el mismo protocolo de irrigación, realizando en la última etapa de la instrumentación, una irrigación final con EDTA, para eliminar el barro dentinario y favorecer la penetración del sellador dentro de los túbulos dentinarios, mejorando así la capacidad de sellado. Los selladores elegidos para el presente estudio fueron un sellador a base de resina epoxica, $\mathrm{AH}$ Plus (Dentsply, Suiza), considerado mundialmente como el sellador "Gold Standard" en endodoncia ${ }^{21}$; el cual fue comparado con el sellador biocerámico Bio-C Sealer (Angelus), el cual fue introducido más recientemente en el mercado, como material biocerámico con propiedades de biocompatibilidad y bioactivo sobre el tejido receptor ${ }^{22}$.

Los hallazgos del presente estudio demostraron que las dimensiones de los conductos difirieron fundamentalmente a la inherente variabilidad anatómica de los molares inferiores evaluados. Esta característica afectó fortuitamente a los valores medios de las dimensiones de los conductos mesiales de esta muestra en ambos grupos, resultandos mayores los tamaños de los conductos en el grupo AH Plus en contraste con el grupo Bio-C Sealer, valores que fueron obtenidos considerando la suma de los volúmenes ocupados por sellador, gutapercha y espacios vacíos.

Al evaluar el porcentaje de volumen ocupado según material, conducto y grupo, no se encontró diferencias significativas, sólo considerando únicamente la categoría de volumen de espacios vacíos, la frecuencia de casos en los cuales pudieron detectarse fue del $15 \%$, o sea 6 casos de 40 , de los cuales 2 se registraron en Bio-C Sealer (5\%) y 4 en AH PLUS (10\%), esto implica un mayor riesgo relativo (RR) de ocurrencia de casos con espacios vacíos al utilizar AH PLUS respecto a Bio-C Sealer. En otras palabras, de acuerdo a la muestra evaluada, la probabilidad de detectar volúmenes de espacios vacíos en conductos obturados con AH PLUS sería el doble que con Bio-C Sealer. Esta última observación, podría deberse a una mayor fluidez del sellador Biocerámico Bio-C Sealer en comparación con el sellador AH Plus ${ }^{20}$, mejor difusión de las partículas

Revista Methodo: Investigación Aplicada a las Ciencias Biológicas. Universidad Católica de Córdoba. Jacinto 
Alison Hernández P, Fernández Bodereau E, Moyano T, Arguelles S, Solla C, Mattea F, Valente M, Martin G. Obturación endodóntica de las raíces mesiales de molares inferiores con sellador Bio-C Sealer y AHPlus.

del sellador en los túbulos dentinarios para producir un enlace mecánico ${ }^{23}$, infiltración del contenido mineral del sellador en la dentina intertubular que resulta en el establecimiento de una zona de infiltración mineral producida después de la desnaturalización de las fibras de colágeno con un sellador altamente alcalino ${ }^{22,24}$.

\section{Conclusión}

Bajo las condiciones del presente estudio, puede concluirse que ambos selladores AH Plus y Bio-C Sealer resultaron adecuados para la obturación de los conductos mesio-vestibulares y mesiolinguales de molares inferiores. Sin embargo, el volumen de espacios vacíos fue menor cuando se usó el sellador biocerámico Bio-C Sealer.

\section{Bibliografía}

1. Schilder H. Filling root canals in three dimensions. Dent Clin North Am 1967; 11:723-44.

2. Spangberg LSW. Instruments, materials, and Devices. In: Cohen S, Burns RC. Pathways of the pulp. 7th ed. St. Louis: Mosby; 1998. p. 476-531.

3. Soares IJ, Goldberg.F. Endodontics Technique and Fundamentals. Editorial Panamericana, Marcelo T de Alvear, Buenos Aires. 2002. P. 152.

4. De Deus QD. Frequency, location, and direction of the lateral, secondary, and accessory canals. J Endod 1975; 1:361-6.

5. Hsu YY, Kim S. The resected root surface. The issue of canal isthmuses. Dent Clin North Am 1997; 41:529-40

6. Hargreaves KM, Cohen S.Pulp roads.Chapter 7: Morphology of the tooth and Preparation of the cavity to the Acces.Frank J. Vertucci, James E. Haddix. Décima edición. 2011 Elsevier, España S.L. Pág. 208-216

7. Hargreaves KM, Cohen S.Pulp Roads.Chapter 10: Obturation of the clean and shaped root canal system. William T. Johnson, James C. Kulild. Décima edición. 2011 Elsevier, España S.L. Pág. 359-362

8. Johnson Wt, Guttmann J. Obturation of cleaned and shaped root canal system. In Cohen S, Hargreaves K. Pathways of the pulp. 9th ed. Philadelphia, PA: Elsevier; 2007.

9. Jitaru S, Hodisan I, Timis L, Lucian A, Bud M.The use of bioceramics in endodontics - literature review. Clujul med. 2016;89(4):470-473

10. Sevimay s, Kalayci a. Evaluation of apical sealing ability and adaptation to dentine oftworesin-basedsealers.J Oral Rehabil. 2005; 32:105-10.

11. Parirokh M, Torabinejad M. Mineral Trioxide Aggregate: An Exhaustive Literature Review - part III: Clinical Applications, Disadvantages and Mechanism of Action. $\mathbf{J}$ Endod. 2010; 36: 400-13.

12. Soheilipour E, Kheirieh S, Madani M, et al. Particle Size of a New Endodontic Cement compared to Root MTA and Calcium Hydroxide. Iran Endod J. 2009; 4: 112-6.

13. Camilleri J.Mineral Trioxide Aggregate: Present and Future Developments. J Endod. 2015; 32 (1): 31-46.

14. Koch K, Brave D, Nasseh A. A Review of Bioceramic Technology in Endodontics. Roots. 2012; 4:6-12.

15. De-deus G, Brandao Mc, Leal F, et al.Lack of Correlation between the Penetration of the Sealant in the Dentinal Tubules and the Sealing Capacity in the Unfetched Root Fillings. Int Endod J. 2012; 45: 642-51

16. Kokkas A, Boutsioukis A, Vassiliadis L, StavrianosCk. The Influence of the smear layer on the Depth of Penetration of the Dentinal Tubules by three Different Radicular Channel Sealants: an in vitro study. J Endod. 2004; 30: 100-2

17. Mamootil K, Messer H.Penetration of the Dentinal Tubules by Endodontic Cements in Extracted Teeth and in vivo. Int Endod $\mathrm{J}$. 2007; 40: 873-81.

18. Wang Z, Shen Y, Haapasalo M.Dentin Extends the Antibacterial Effect of Endodontic Sealers against the Enterococcus faecalis Biofilms. J Endod. 2014; 40: 505-8.

19. Cheng 1, Ye F, Yang R, Lu X, Shi Y, Li 1, et al.Osteoinduction of hydroxyapatite/betatricalcium phosphate bioceramics in mice with a fractured fibula. Acta biomater. 2010; 6 (4): 1569- 1574.

20. Candeiro GT, Correia FC, Duarte MA, Ribeiro-Siqueira DC, Gavini G. Evaluation of radiopacity, $\mathrm{pH}$, release of calcium ions, and flow of a bioceramic root canal sealer. J Endod. 2012;38(6):842-5. Epub 2012/05/19. doi: 10.1016/j.joen.2012.02.029. 
21. Resende LM, Rached-Junior FJ, Versiani MA, Souza-Gabriel AE, Miranda CE, SilvaSousa YT, et al. Estudio comparativo de las propiedades fisicoquímicas de los selladores del conducto radicular AH plus, epifanía y epifanía SE. Int Endod J. 2009; 42: 785-93.

22. L. Han and T. Okiji, "Uptake of calcium and silicon released from calcium silicate-based endodontic materials into root canal dentine," International Endodontic Journal, vol. 44, no. 12, pp. 1081-1087, 2011.

23. Zhang W, Li Z, Peng B. Assessment of a new root canal sealer's apical sealing ability. Oral Surg Oral Med Oral Pathol Oral RadiolEndod. 2009;107(6): e79-82. Epub 2009/05/26. doi: 10.1016/j.tripleo.2009.02.024.

24. A. R. Atmeh, E. Z. Chong, G. Richard, F. Festy, and T. F. Watson, "Dentin-cement interfacial interaction: calcium silicates and polyalkenoates," Journal of Dental Research, vol. 91, no. 5, pp.454-459, 2012.

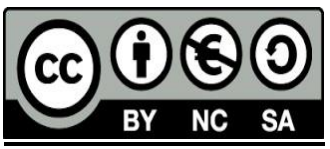

